\title{
La seguridad social en México a dos décadas de las reformas privatizadoras. Balance y perspectivas
}

\section{Social security in Mexico after two decades of privatization reforms. Balance and perspectives}

doi: http://dx.doi.org/10.32870/

espiral.v25i73.6247

\begin{abstract}
Resumen
Este artículo presenta un análisis de la situación que guarda la seguridad social en México a dos décadas de implementación de las reformas que originaron el proceso de privatización e individualización de los sistemas de pensiones. Se hace un balance de los beneficios que se atribuían a su implementación, así como de las condiciones económicas y políticas que existen para avanzar hacia la universalización de la previsión social en el país. La evidencia demuestra resultados decepcionantes: la situación financiera de los institutos sigue siendo precaria, su cobertura es limitada y las pensiones representan una cuarta parte del salario promedio cotizado. Lo más preocupante es que se insista en aplicar las mismas medidas (elevar contribuciones, reducir beneficios y mercantilizar la operación del sistema), ofreciendo a cambio acceso universal a una pensión mínima y a un incipiente seguro de desempleo.
\end{abstract}

Palabras clave: previsión social, universalización, privatización, institutos de seguridad social, México.

\author{
Gerardo Ordóñez Barba* \\ Miguel Ángel Ramírez Sánchez $*$
}

\begin{abstract}
This paper presents an analysis of the situation of social security in Mexico after two decades of the reforms that led to the process of privatization and individualization of pension systems. It makes a balance of the benefits attributed to its implementation, as well of the existing economic and political conditions that move towards the universalization of social welfare in the country. The evidence shows disappointing results: the financial situation of the institutes remains precarious, their coverage is limited, and pensions represent a quarter of the average salary quoted.What worries the most is the insistence on applying the same measures (raise contributions, reduce benefits, and commercialize the operation of the system), offering in exchange universal access to a minimum pension and to an incipient unemployment insurance.
\end{abstract}

Keywords: Social welfare, universalization, privatization, social security institutes,

Mexico.

\footnotetext{
-Profesor-Investigador del Colegio de la Frontera Norte (Colef), México. ORCID: 0000-00033498-5808_ordonez@colef.mx

• Profesor de la Universidad Autónoma de Baja California, México. ORCID: 0000-000 I-60098482 miguel.angel.ramirez.sanchez@uabc.edu.mx

Fecha de recepción: 13 de octubre de 2016. Fecha de aceptación: 09 de abril de 2018.
} 


\section{Introducción}

En este trabajo se presenta un balance de la situación que guarda el sistema mexicano de seguridad social, creado en 1943. Se revisa lo que queda en pie del mismo después de dos décadas de reformas inspiradas en el llamado consenso de Washington, incluyendo la transferencia al sector privado de los fondos de pensiones que hasta 1997 y 2006 habían administrado las instituciones públicas de seguridad social. Se realiza un enfoque en tres puntos clave para el futuro del sistema: la ya mencionada privatización de los fondos de pensiones, la situación financiera de los principales institutos de seguridad social y las propuestas para universalizar la protección social que se discuten ahora, incluyendo el paquete de iniciativas legislativas que el actual presidente de la república (2012-2018) sometió en 2013 al Congreso de la Unión para crear un sistema universal de seguridad social.

Dado el carácter inacabado de este último intento de reforma, el trabajo se concentra en la frustrada iniciativa presidencial de creación de un seguro de desempleo, aprobada por la Cámara de Diputados en 2015 y pendiente de trámite desde entonces por parte de la Cámara de Senadores. Este último punto permite destacar el carácter incompleto del sistema, considerando que México es el único país de la Organización para la Cooperación y el Desarrollo Económicos (OCDE) cuyos habitantes no cuentan con seguro de desempleo. Su falta de aprobación en el Senado demuestra la oposición que existe entre los partidos políticos en torno a la reforma.

Muchos autores han señalado las inconsistencias del discurso de los reformadores, que insisten en presentar los cambios que proponen como indispensables, inevitables, urgentes y definitivos, aunque cada cierto tiempo anuncian la necesidad de una nueva ronda de reformas (Valencia y Ordoñez, 2016). Como se demuestra en este trabajo, los 
problemas denunciados por los reformadores existen, pero han sido exagerados, sus verdaderas causas se han distorsionado, sus posibles soluciones se han idealizado, los costos de la transición se han soslayado y pocas veces se ha asumido el fracaso de medidas que resultaron contradictorias.

Para desarrollar los objetivos planteados, se ha dividido el artículo en cuatro apartados. El primero se dedica a explicar la reforma de las pensiones, que es el cambio más importante del sistema mexicano de seguridad social en los últimos treinta años, y quizá en toda su historia. Con estas reformas, se remplazó un esquema de pago intergeneracional, en el que los trabajadores activos pagaban las pensiones de los retirados, por un esquema individualizado de ahorro mediante el cual los trabajadores activos ahorran para su retiro durante su vida laboral. Sin embargo, como se documenta, el nuevo esquema paga pensiones de montos inferiores a los esperados y produce un costo fiscal demasiado alto, es decir, un costo de transición que apenas fue considerado cuando se discutían las supuestas bondades del cambio.

En el segundo apartado, se revisa la situación financiera que guardan los institutos de seguridad social en el país, luego de perder el manejo de los fondos de pensiones. Como se esperaba, la falta de esos fondos está siendo compensada con mayores transferencias del Gobierno federal, aunque este costo tampoco se discutió suficientemente mientras se preparaban las reformas. En este punto, se ha señalado que el problema mayor está en los seguros de salud que aún conservan los institutos de seguridad social, los cuales operan con grandes déficits por la renuncia del Gobierno y la oposición de los patrones a fijar una cuota que cubra totalmente sus costos de operación.

En el tercer apartado, se discute la propuesta de universalizar la seguridad social que impulsa el actual Gobierno federal. 
Finalmente, en el último apartado, se enlistan las principales conclusiones y se ponen sobre la mesa las desalentadoras perspectivas que se vislumbran para el problema de estudio de mantenerse en la ruta de las reformas privatizadoras iniciadas hace veinte años.

\section{I.Privatización de los fondos de pensiones}

Desde 1995, el sistema mexicano de seguridad social es objeto de reformas que han modificado profundamente el modelo de pensiones que el país adoptó desde la creación del Instituto Mexicano del Seguro Social (IMSS), en 1943. Hasta el momento, se han reformado los esquemas de pensiones del IMSS en 1995, del Instituto de Seguridad y Servicios Sociales para los Trabajadores del Estado (ISSSTE) en 2003, de la mayoría de los estados entre 2000 y 2009, de varias universidades públicas a partir de 2013, y de la Comisión Federal de Electricidad (CFE) y Petróleos Mexicanos (PEMEX) en 2015.

Con estas reformas, la federación se propuso romper con el régimen de solidaridad intergeneracional (también llamado de reparto o de bolsa común), mediante el cual la población activa pagaba las pensiones de los retirados, quienes usualmente recibían un ingreso equiparable a su último salario (beneficio definido). De acuerdo con la postura oficial, la aplicación de estas reformas resultaba indispensable y hasta urgente por la carencia de reservas actuariales y la poca relación entre contribuciones y beneficios, lo que había provocado que la mayoría de los planes de pensiones presentaran serios desequilibrios y fueran financieramente inviables (Auditoría Superior de la Federación, 2014).

En respuesta al deterioro financiero de estos planes, el Gobierno federal decidió intervenir y modificar sus bases de operación. El objetivo central fue sustituir el viejo esquema de reparto por el Sistema de Ahorro para el Retiro (SAR), estableciendo que las aportaciones a los fondos de pensio- 
nes fueran depositadas en cuentas individuales únicas, las cuales se supone generarán, a lo largo de la vida productiva del trabajador y con la administración de entidades privadas, denominadas Administradoras de Fondos para el Retiro (AFORES), los recursos necesarios para el sostenimiento de cada pensionado.

Este fue, sin duda, un cambio de fondo, considerando que el SAR rompe con el principio de solidaridad y hace depender la pensión de cada individuo de los recursos acumulados en su cuenta personal. Después de su desaparición a la vista de todos, puede decirse que el principio de solidaridad apenas sobrevive focalizado en la forma de planes asistenciales para los excluidos del sistema y diluido en la forma de una pensión mínima garantizada para aquellos que contribuyeron al sistema pero no lo hicieron en la cantidad necesaria para obtener un ingreso mínimo para la supervivencia.

Al cambio descrito se le atribuyen cualidades de muy diverso tipo, incluyendo su capacidad de mejorar las condiciones de protección social, aunque esto sólo inicialmente, pues en la medida que esta promesa ha resultado imposible de cumplir, el énfasis se ha puesto en el alivio para las finanzas públicas que significan los recortes hechos a los pasivos laborales de las instituciones de seguridad social y en los beneficios que el nuevo esquema tiene para la economía nacional.

También, entre otras cosas, se ha dicho que el esquema de contribuciones definidas permite aumentar gradualmente el volumen del ahorro interno y su disponibilidad para las necesidades de la producción. No obstante, los fondos radicados en las entidades privadas han regresado a las arcas gubernamentales convertidos en deuda pública interna, lo que representa un serio obstáculo para cumplir con su propósito de apoyar a las actividades productivas. Por otra parte, en lo que se refiere a los efectos del nuevo esquema sobre los montos de las pensiones y de las presta- 
ciones económicas derivadas de otros riesgos, los cambios han tenido efectos contraproducentes, y está claro que las condiciones para tener derecho a estos ingresos son ahora más restrictivas.

\section{I.I Reformas a la Ley del Seguro Social (1995) y la ley del ISSSTE (2007)}

En las dos mayores instituciones del sistema, el IMSS y el ISSSTE, las reformas descritas aumentaron la edad de retiro y el tiempo de cotización necesario para obtener una pensión. En el caso del IMSS, la ley promulgada en 1995 aumentó las semanas de cotización necesarias de quinientas a 1 250. Además, en la pensión por cesantía avanzada se incluyó el requisito de edad mínima de sesenta años, que no existía en la antigua ley (ver: Tabla 1).

Tabla 1. Cambios al plan de pensiones de trabajadores que cotizan en el IMSS

Ley del IMSS de 1973 (reformada en 1992)

Esquema de beneficios definidos

Seguro de invalidez, vejez, cesantía en edad avanzada y muerte (IVCEAM)

Tipos de pensiones y requisitos de acceso

- Vejez: quinientas semanas de cotización y sesenta y cinco años de edad

- Cesantía en edad avanzada: quinientas semanas de cotización - Invalidez: ciento cincuenta semanas de cotización

- Muerte del asegurado: ciento cincuenta semanas de cotización

\section{Ley del IMSS de 1995}

Esquema de contribuciones

definidas

Seguro de retiro, cesantía y muerte (RCM)

Tipos de pensiones y requisitos de acceso

- Vejez: 1250 semanas de cotización y sesenta y cinco años de edad

- Cesantía en edad avanzada: 1250 semanas de cotización y sesenta años de edad 


\begin{tabular}{|c|c|}
\hline Beneficios por tipo de pensión & $\begin{array}{l}\text { Beneficios para ambos tipos de } \\
\text { pensiones }\end{array}$ \\
\hline $\begin{array}{l}\text { - Invalidez y vejez: un porcentaje } \\
\text { del salario promedio de los últimos } \\
\text { cinco años, más una fracción por } \\
\text { cada año en exceso a los primeros } \\
\text { diez. La pensión esta indizada a } \\
\text { los incrementos del salario mínimo } \\
\text { general y no puede ser mayor } \\
\text { al último salario que recibió el } \\
\text { trabajador } \\
\text { - Cesantía: de } 75 \% \text { a } 95 \% \\
\text { de la pensión que le hubiera } \\
\text { correspondido de cumplir sesenta y } \\
\text { cinco años } \\
\text { - Muerte del asegurado: un } \\
\text { porcentaje de la pensión por } \\
\text { invalidez, vejez o cesantía en } \\
\text { edad avanzada que le hubiera } \\
\text { correspondido al asegurado, de } 90 \% \\
\text { en el caso de su viuda y de } 20 \% \\
\text { en el caso de sus hijos de dieciséis } \\
\text { a veinticinco años de edad, o sus } \\
\text { padres cuando el asegurado no } \\
\text { tuviera hijos de la edad consignada } \\
\text { ni cónyuge }\end{array}$ & $\begin{array}{l}\text { El saldo acumulado en cuenta } \\
\text { de AFORES más rendimientos } \\
\text { obtenidos por inversiones de la } \\
\text { Sociedad de Inversión de Fondos } \\
\text { para el Retiro (SIEFORE) }\end{array}$ \\
\hline Pensión mínima garantizada & Pensión mínima garantizada \\
\hline $\begin{array}{l}\text { Equivalente al } 90 \% \text { del salario } \\
\text { mínimo general, indizado con el } \\
\text { aumento anual al salario mínimo } \\
\text { general }\end{array}$ & $\begin{array}{l}\text { Equivalente al } 100 \% \text { del salario } \\
\text { mínimo general del } 1 \text { de julio } \\
\text { de } 1997 \text {, indizado con el Índice } \\
\text { Nacional de Precios al Consumidor } \\
\text { (INPC). Equivale a MXN } 2707.31 \text { al } \\
1 \text { de febrero de } 2016\end{array}$ \\
\hline
\end{tabular}


Aportaciones para el seguro de invalidez, vejez, cesantía en edad avanzada y muerte (IVCM) (SBC): $8.750 \%$ por la empresa, $3.125 \%$ por el trabajador, y por parte del Estado $7.143 \%$ de la cuota patronal
Aportaciones para el seguro por

retiro, cesantía y vejez (RCV)

$6.5 \%$ del sBC, más cuota social

Para la cuenta de retiro: $2.0 \%$ por la empresa; para las cuentas de cesantía en edad avanzada y vejez: $3.150 \%$ por la empresa, $1.125 \%$ por el trabajador y $7.143 \%$ de la cuota del patrón por parte del Estado. A lo anterior, se suma una cuota social a cargo del Gobierno federal de MXN 4.38 diarios en promedio por trabajador, aplicada en forma progresiva de acuerdo a rangos de salarios mínimos. El Instituto Mexicano del Seguro Social (2015) calcula que la suma de aportaciones y cuota social equivale, en promedio, al $7.96 \%$ del SBC. Dado que la cuota social varía según el nivel de ingreso, la aportación total para trabajadores que ganan un salario mínimo es de $13.606 \%$ del SBC, y para los trabajadores que ganan diez salarios mínimos de $7.122 \%$ 
Generación de transición

Los trabajadores en servicio antes del 1 de julio de 1997, fecha de entrada en vigor de la reforma, pueden decidir pensionarse bajo los términos de la ley de 1973. En el momento de su retiro, estos trabajadores pueden elegir entre: 1) los beneficios de la ley de 1973, más los fondos que les correspondan por las contribuciones al retiro de 1997 hasta la fecha de retiro; o 2) el saldo acumulado en las AFORES desde 1997, más el saldo que les corresponda por las aportaciones al SAR y al Instituto del Fondo Nacional de Vivienda para los Trabajadores (INFONAVIT) por el periodo 1992-1997

Fuentes: elaboración propia con información de Instituto Mexicano del Seguro Social (1992, 1995, 2015), Morales (2007), Secretaría del Trabajo y Previsión Social (1973), Organización para la Cooperación y el Desarrollo Económicos (2016) y Villagómez (2015).

En el caso del Issste, la ley de 2007 aumentó la edad de retiro de cincuenta y cinco a sesenta y cinco años de edad, y el tiempo de cotización de quince a veinticinco años. Para las pensiones por vejez y por cesantía en edad avanzada, el aumento del tiempo de cotización fue de diez a veinticinco años. Cambios similares se han producido en otros fondos de pensiones reformados. 


\section{Tabla 2. Cambios al plan de pensiones de trabajadores que cotizan en el ISSSTE}

Ley del ISSSTE de 1983

Esquema de beneficios definidos

Seguros de jubilación, de retiro por edad y tiempo de servicios, y cesantía en edad avanzada

Tipos de pensiones y requisitos de acceso

- Vejez, jubilación o retiro: treinta años de cotizaciones (1 500 semanas) para los hombres y veintiocho años (1 400 semanas) para las mujeres - Cesantía en edad avanzada: sesenta años de edad y diez años de cotizaciones (quinientas semanas)

- Retiro por edad y tiempo de servicios: cincuenta y cinco años de edad y quince años de cotizaciones (setecientas cincuenta semanas)

Beneficios por tipo de pensión

Vejez, jubilación o retiro: $100 \%$ del promedio del sueldo base del último año, con un máximo de diez salarios mínimos generales vigentes en el Distrito Federal (SMGVDF) Retiro por edad y tiempo de servicios: igual a la pensión por jubilación, reducida hasta 50\% por los años de antigüedad que falten para completar treinta
Ley del ISSSTE de 2007

Esquema de contribuciones definidas

Seguro por RCV

Tipos de pensiones y requisitos de acceso

- Vejez y cesantía en edad avanzada: sesenta años de edad y veinticinco años de cotizaciones (1 250 semanas)

- Retiro: sesenta y cinco años de edad y veinticinco años de cotizaciones (1 250 semanas)

Beneficios para ambos tipos de pensiones

El saldo acumulado en cuenta de AFORES más rendimientos obtenidos por inversiones de SIEFORES

\section{Pensión mínima garantizada}

Equivale a dos veces el salario mínimo vigente al momento de expedirse la ley, actualizado por el INPC 


\begin{tabular}{|c|c|}
\hline Aportaciones para los seguros & Aportaciones para el seguro $R C V$ \\
\hline de jubilación, pensiones $e$ & \\
\hline indemnización global & $11.3 \%$ del sBC: $6.125 \%$ por la \\
\hline & dependencia y $6.125 \%$ por el \\
\hline 7.0\% del SBC: $3.5 \%$ por la dependencia & trabajador. La contribución está \\
\hline y $3.5 \%$ por el trabajador & limitada a diez veces el SMGVDF, \\
\hline & más cuota social pagada por \\
\hline & Gobierno federal \\
\hline & Generación de transición \\
\hline & Los trabajadores activos al \\
\hline & momento de ser aprobada la \\
\hline & reforma pudieron elegir, hasta el \\
\hline & 15 de agosto de 2008 , cambiar al \\
\hline & nuevo sistema o permanecer con el \\
\hline & antiguo, pero con reglas diferentes. \\
\hline & De 2010 a 2028, el requisito de edad \\
\hline & aumenta paulatinamente hasta \\
\hline & llegar a los siguientes parámetros: \\
\hline & 1) Pensiones de jubilación. Hombres: \\
\hline & sesenta años de edad y treinta años \\
\hline & $\begin{array}{l}\text { de servicios. Mujeres: cincuenta y } \\
\text { ocho años de edad y veintiocho años }\end{array}$ \\
\hline & de servicio \\
\hline & 2) Pensión de retiro por edad y \\
\hline & tiempo de servicio. Sesenta años de \\
\hline & edad y quince años de cotizaciones \\
\hline & 3) Pensión por cesantía en edad \\
\hline & avanzada. Sesenta y cinco años de \\
\hline & edad y diez años de cotizaciones \\
\hline & Los trabajadores que optaron por \\
\hline & el nuevo sistema recibieron un \\
\hline & bono de reconocimiento de derechos \\
\hline & en el esquema anterior. Su valor \\
\hline & fue equivalente a la pensión a que \\
\hline & hubieran tenido derecho al cierre \\
\hline & de 2006 \\
\hline
\end{tabular}

Fuentes: elaboración propia con información de Instituto de Seguridad y Servicios Sociales de los Trabajadores del Estado (1983, 2007), Morales (2007) y Villagómez (2015). 


\section{I.2 Resultados decepcionantes}

En términos generales, los resultados del nuevo modelo son decepcionantes. El SAR no logra una tasa adecuada de remplazo al salario y se estima que las pensiones futuras estarán en su mayoría en el nivel mínimo garantizado (Ham, 2000, p. 613). En su más reciente informe sobre las pensiones en México, la OCDE (Organización para la Cooperación y el Desarrollo Económicos, 2016, p. 3) califica al sistema como "un éxito en cuanto a que ha aumentado la capacidad de la economía mexicana para financiar las pensiones", y destaca los montos acumulados en las AFORES (14.1\% del producto interno bruto -PIB-) y los rendimientos que ofrecen (6.2\%). Sin embargo, el mismo informe reconoce que el nuevo modelo está amenazado por el desencanto y el desprestigio: en el mejor de los casos, contribuciones al SAR del $6.5 \%$ del salario base significan pensiones del 26\% del salario promedio cotizado (Organización para la Cooperación y el Desarrollo Económicos, 2016).

Para evitar el previsible colapso del sistema, la OCDE recomienda aumentar los requisitos para acceder a una pensión y recortar aún más sus beneficios. El organismo considera que es urgente ajustar cuatro parámetros: 1) bajar el monto máximo de las pensiones de veinticinco a diez veces el salario mínimo, 2) retrasar la edad de retiro, 3) aumentar el monto de las cotizaciones y 4) promover el ahorro voluntario.

También, estima que para lograr una tasa de reemplazo de $50 \%$, con una probabilidad de $75 \%$ a $90 \%$, los trabajadores deberían aportar en promedio de $13 \%$ a $18 \%$ de su salario durante cuarenta años (contra los diez años que exigía la ley del IMss de 1973 y los veinticinco de la de 1995), y recomienda seguir reformando los planes de pensiones de reparto que aún se mantienen en pie para reducir la carga del sistema. 
Con el mismo propósito, la OCDE propone reducir la pensión de los trabajadores que permanecen en esquemas transitorios de beneficios definidos. Esta última propuesta afectaría a unos diez millones de trabajadores del sector privado y 1.6 millones del sector público que comenzaron a trabajar y cotizar antes de las reformas a las leyes del IMSS ${ }^{1}$ y el ISSSTE, ${ }^{2}$ lo que les permite retirarse bajo los términos de las leyes anteriores, que contemplan pensiones de hasta el 100\% del último salario. Curiosa definición de éxito tiene la OCDE: menos beneficios y más requisitos. ${ }^{3}$

$\mathrm{Ni}$ en sus mejores momentos, la cobertura conjunta de los programas de pensiones del IMSS, ISSSTE, PEMEX, el Instituto de Seguridad Social para las Fuerzas Armadas Mexicanas (ISSFAM) y la extinta Compañía de Luz y Fuerza del Centro (CLYFC) superó nunca al 40\% de la población eco-

I. La ley del IMSS de 1995 reconoció a los trabajadores en servicio antes del I de julio de 1997 el derecho a que, llegado el momento de retirarse, pudieran hacerlo bajo los términos de la ley del instituto de 1973. Esta garantía cubrió a los 10.2 millones de trabajadores que el IMSS afiliaba en mayo de 1997.

2. Un artículo transitorio de la nueva ley del ISSSTE, el décimo, dispuso que todos los trabajadores en activo al 3 I de marzo de 2007, que Murillo y Venegas (20I I) estiman en 2.07 millones, podrían elegir entre el anterior sistema de reparto, con algunas modificaciones, y el nuevo sistema de cuentas individuales. La inmensa mayoría se decidió por el anterior sistema. Sólo el $14 \%$ de trabajadores públicos optaron por las cuentas individuales (Organización para la Cooperación y el Desarrollo Económicos, 2016, p. 49). En 2013, I 646000 trabajadores en activo cotizaban en el plan de pensiones amparado por el décimo transitorio (Auditoría Superior de la Federación, 20I4) y, hasta el año 20l4, un total de 408000 pensionados habían accedido a este derecho por la misma vía (Instituto de Seguridad y Servicios Sociales de los Trabajadores del Estado, 20I6).

3. Las recomendaciones de la OCDE de una nueva reforma para complementar la anterior recuerdan la última fase del ciclo de vida de las reformas pro consenso de Washington, que Valencia identificó en las reformas mexicanas de los últimos treinta años: "Las fases de este ciclo pueden ser caracterizadas de manera sintética de la siguiente manera:identificación de la necesidad de reforma, diseño reformista, búsqueda de legitimación nacional e internacional ante la opinión pública y actores sociales, políticos y económicos, superación de obstáculos (generación de aliados e identificación de opositores), formalización (a través de un proceso legislativo o de la creación de un decreto presidencial), celebración y difusión de las nuevas expectativas, [e] incorporación e institucionalización en la vida política y económica cotidiana hasta la identificación de la necesidad de una nueva reforma para complementar la anterior" (Valencia, 20I6, pp. II-I2). 
nómicamente activa (PEA) (Solís y Villagómez, 1999, p. 112). Cálculos de la Auditoria Superior de la Federación (ASF) estimaban, para el año 2013, en 20.9 millones el número de trabajadores que cotizaban para recibir una pensión en su vejez. Esta cifra representa el 39.7\% de la PEA y el $41.6 \%$ de la población ocupada, una cobertura insuficiente bajo cualquier criterio de medida. Los incrementos en la cobertura de los últimos años (2011-2013) son insignificantes, del orden del $2 \%$ anual. A este ritmo, se tendría que esperar hasta el año 2060 para ver a toda la PEA enrolada en un plan de pensión contributivo, pero aun en ese escenario es posible que en el camino aparezcan dificultades insalvables que impidan llegar a la meta. El tamaño del empleo informal sugiere que el sistema está ya muy cerca de su máxima cobertura posible.

Tabla 3. Cobertura del sistema de pensiones en México (2010-2013)

(cifras en millones de personas)

\begin{tabular}{lrrrr} 
Concepto & 2010 & 2011 & 2012 & 2013 \\
I. PEA & 48.7 & 51.2 & 51.6 & 52.6 \\
II. Población ocupada & 46.1 & 48.7 & 49.1 & 50.2 \\
III. Trabajadores con acceso a plan de pensión & 18.1 & 18.1 & 18.6 & 20.9 \\
$\quad$ ISSSTE & 2.6 & 2.7 & 2.7 & 2.8 \\
IMSS & 14.6 & 15.2 & 15.9 & 16.5 \\
ISSFAM & 0.5 & $\mathrm{nd}$ & $\mathrm{nd}$ & 1.1 \\
$\quad$ Banco de México & $\mathrm{ns}$ & $\mathrm{ns}$ & $\mathrm{ns}$ & $\mathrm{ns}$ \\
Universidades públicas estatales & 0.1 & $\mathrm{nd}$ & $\mathrm{nd}$ & 0.1 \\
$\quad$ PEMEX & 0.1 & $\mathrm{nd}$ & $\mathrm{nd}$ & 0.1 \\
$\quad$ Sociedades nacionales de crédito & $\mathrm{ns}$ & $\mathrm{ns}$ & $\mathrm{ns}$ & $\mathrm{ns}$ \\
$\quad$ Trabajadores independientes incorporados & 0.2 & 0.2 & $\mathrm{nd}$ & 0.3 \\
en el SAR & & & & \\
IV. Trabajadores sin acceso a plan de pensión & 28.0 & 30.6 & 30.5 & 29.3 \\
Cobertura efectiva (III / I) & $37.2 \%$ & $35.4 \%$ & $36.0 \%$ & $39.7 \%$ \\
Cobertura ocupacional (III / II) & $39.3 \%$ & $37.2 \%$ & $37.9 \%$ & $41.6 \%$ \\
\hline
\end{tabular}

ns: no significativo; nd: no hay dato.

Fuente: Auditoría Superior de la Federación (2014, p. 74). 


\section{I.3 La informalidad, barrera infranqueable}

La debilidad mayor del sistema reformado de pensiones radica en el pequeño tamaño de la economía formal. La informalidad deja sin seguridad social a la mayoría de la población. Este problema explica la gran cantidad de cuentas inactivas que arrastra el SAR. Al 31 de diciembre de 2013, el acumulado de trabajadores con una cuenta AFORE era de 50.8 millones (Auditoría Superior de la Federación, 2014). Esta cifra está muy cerca de los 52.7 millones de la PEA total de 2013 (Instituto Nacional de Estadística y Geografía, 2014, p. 1), y muy lejos de los 17.8 millones de trabajadores en régimen de contribución definida estimables con datos de la ASF para el mismo año (Auditoría Superior de la Federación, 2014). Aunque se trata de cifras acumuladas desde 1998, cuando se abrieron las primeras cuentas, de modo que posiblemente un número indeterminado de estos cincuenta millones haya fallecido o ya disfrute de una pensión y ha dejado de pertenecer a la PEA, la diferencia es muy grande.

Según el censo de población de 2010, el número de personas mayores de sesenta y cinco años que reciben ingreso de una pensión es de apenas 1.27 millones (Instituto Nacional de Estadística y Geografía, 2010). Lo que en realidad explica la diferencia observada es que la mayoría de estas cuentas están inactivas. De acuerdo con datos de la Comisión Nacional del Sistema de Ahorro para el Retiro (CONSAR), se estima que sólo 43.8\% de las cuentas estaban activas durante el cuarto trimestre de 2013 (Comisión Nacional del Sistema de Ahorro para el Retiro, 2014, pp. 16 y 64). ${ }^{4}$ Trabajadores que en algún momento de su vida activa abrieron una cuenta en el SAR dejaron de cotizar por

4. El dato se obtiene de dividir el total de cuentas activas, es decir, "Cuentas con aportación a retiro, cesantía en edad avanzada y vejez (RCV) en los últimos tres años" (Comisión Nacional del Sistema de Ahorro para el Retiro, 2014, p. 64) entre el "Total de cuentas administradas por las AFORES" (Comisión Nacional del Sistema de Ahorro para el Retiro, 2014, p. 16). 
haber perdido su empleo o porque después de haberlo perdido se refugiaron en actividades informales sin derecho a seguridad social. Estas cifras revelan que la informalidad laboral está menguando los fondos de pensiones, desactivando las cuentas registradas y al tiempo inflando el costo fiscal de las pensiones en curso, esto último porque llegado el momento de su retiro estos trabajadores podrían exigir el pago de una pensión mínima garantizada a la que tiene derecho cualquiera con una cuenta en el SAR que hubiera cotizado en algún momento de su vida activa.

\section{I.4 Insolvencia del sistema mexicano de pensiones}

Los cambios no han resuelto el problema de insolvencia que padece el sistema. De acuerdo con valuaciones actuariales de la ASF, en los próximos cien años se requerirán 9.1 billones de pesos, que significan el 69.0\% del PIB de 2013, para cubrir el pago de los setenta y cuatro regímenes públicos de beneficios definidos, a cargo del Gobierno federal, que aún subsisten (Auditoría Superior de la Federación, 2014, p. 136). La ASF estima ahorros para el periodo 2020-2050 del orden de 31.8 billones de pesos de 2013 por el cambio al esquema de contribuciones definidas, pero dicho cambio obligó al Gobierno federal a asumir el costo del traspaso de un esquema a otro.

Por una parte, el Gobierno asumió las pensiones en curso de pago al momento de las reformas (con un valor presente de MXN 146000 millones para el caso del IMSS y MXN 889000 millones en el caso del ISSSTE) y, más importante, asumió la obligación de pagar las pensiones de los trabajadores que decidieron permanecer en esquema de beneficios definidos. El valor presente de las obligaciones de pago de pensiones del régimen del artículo 10 transitorio de la ley del ISSSTE es de dos billones ciento setenta y cinco mil millones de pesos, el más grande de los pasivos laborales, equivalente al $23.7 \%$ del total. 


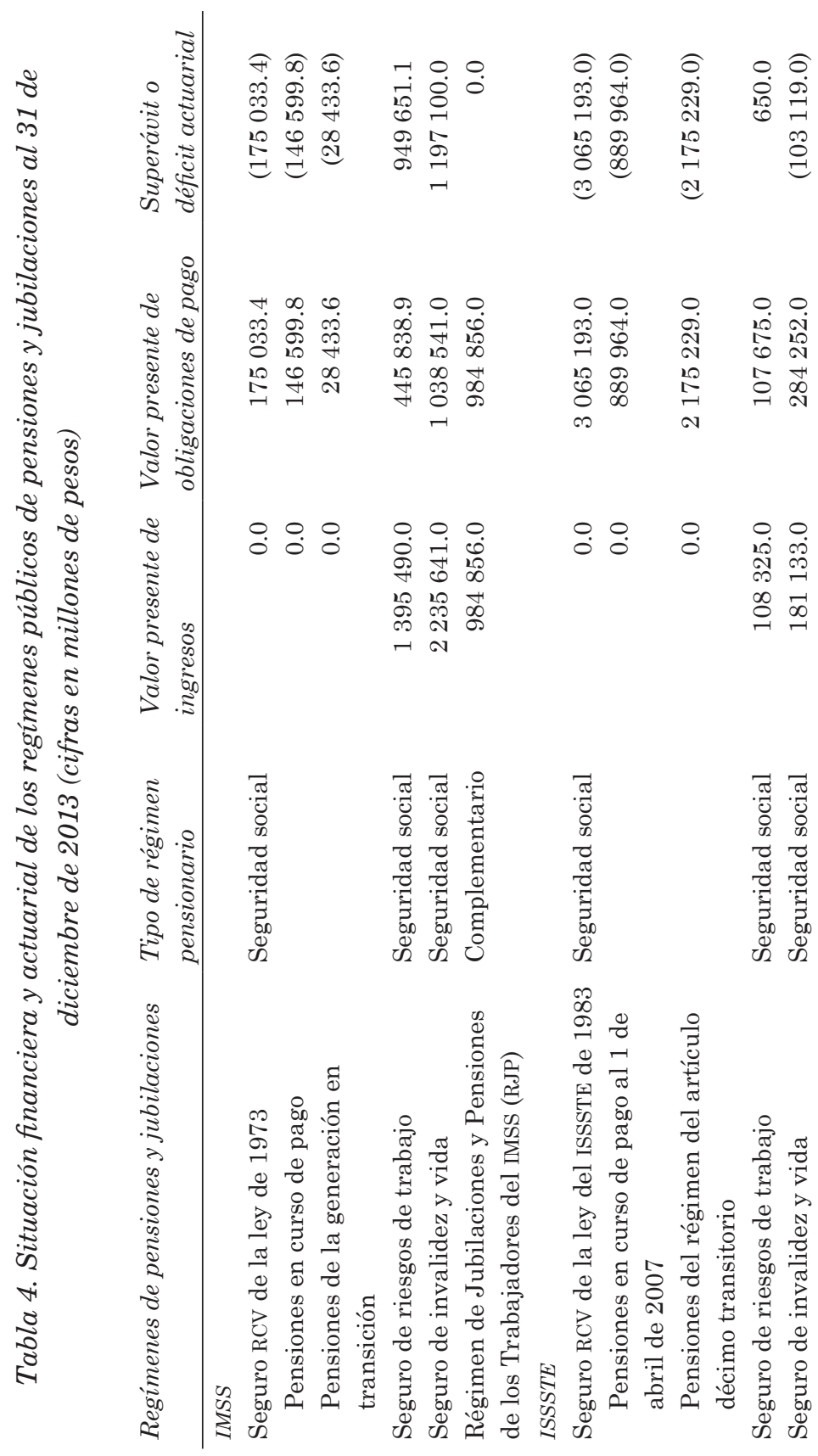




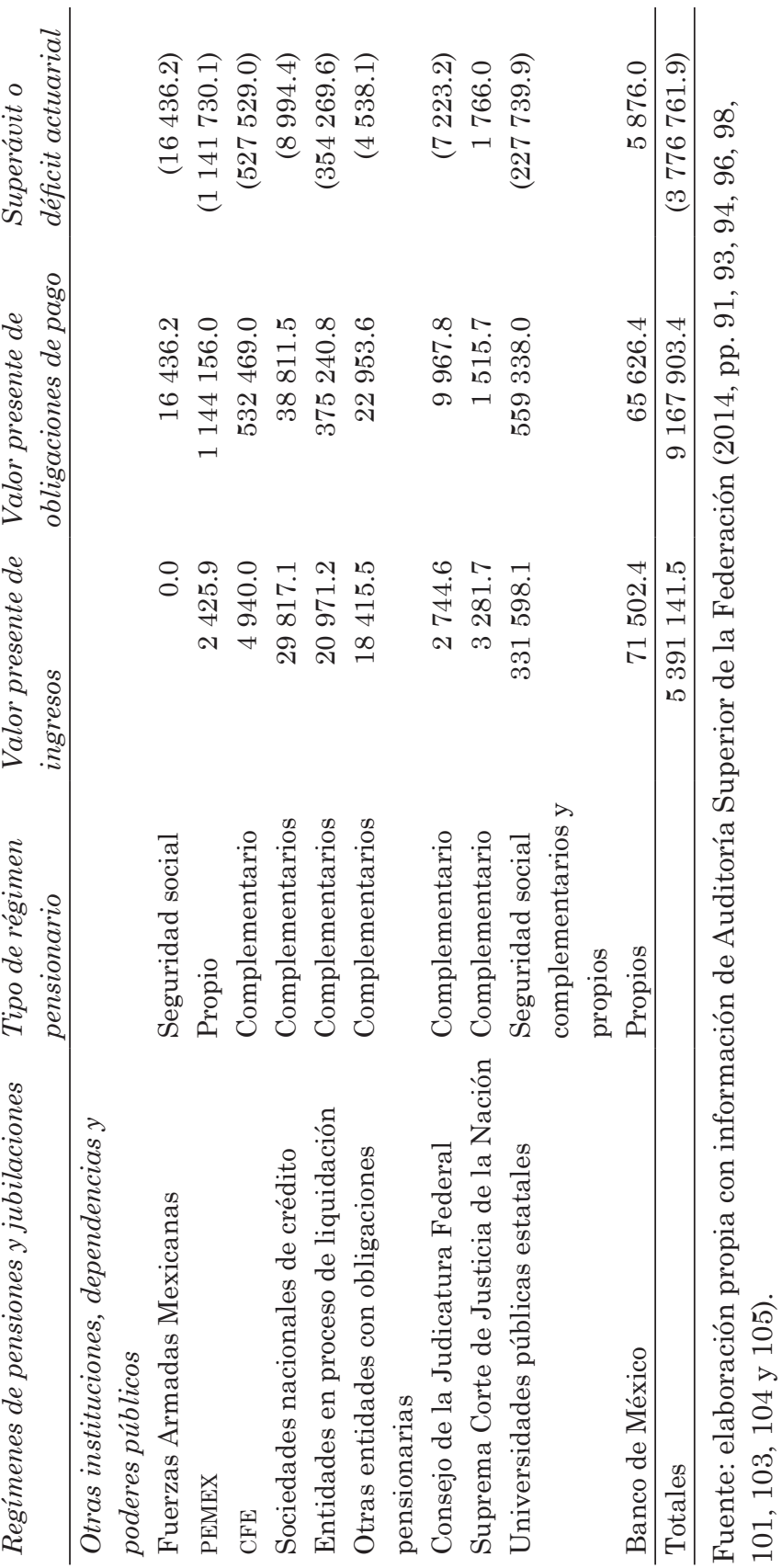


Otro de los costos de las reformas es una bomba de tiempo. La ASF no incluye en sus cálculos el pago de las pensiones de los trabajadores que cotizan en el IMSs que en los próximos años pueden decidir retirarse de acuerdo a la ley de 1971, que les ofrece hasta el $100 \%$ de su salario actual, muy por encima del $26 \%$ que prometen las AFORES. Por otra parte, la baja tasa de remplazo de los planes de AFORE significa que el Gobierno federal deberá desembolsar la diferencia entre la pensión recibida y una pensión mínima garantizada. La ASF estima que para el mismo periodo de 2020-2050, el Gobierno federal deberá desembolsar por este motivo 5.1 billones de pesos de 2013.

\section{I.5 Planes asistenciales}

Una iniciativa que compensa en parte las deficiencias de los planes contributivos de pensiones es la de los planes asistenciales que han establecido el Gobierno federal y algunos locales, como el de la Ciudad de México. Por sus números, el más grande de estos programas es Pensión para Adultos Mayores (PAM), de la Secretaría de Desarrollo Social (SEDESOL), que otorga un ingreso de MXN 580.00 mensuales a personas de sesenta años o más, mexicanos por nacimiento, con un mínimo de veinticinco años de residencia en el país y que no reciban pensión mayor a MXN 1092.00 mensuales por concepto de jubilación o pensión de tipo contributivo. Al sexto bimestre de 2014, el número de personas adultas mayores apoyadas por el programa ascendía a 5.5 millones, una cifra que superaría incluso al número de pensionados del IMSS y del ISSSTE juntos. 
Tabla 5. Pensionados en planes contributivos y asistenciales seleccionados (2014)

\begin{tabular}{lc} 
Institución o programa & Número de pensionados \\
IMSS & 3588964 \\
Asegurados pensionados & 3337397 \\
Extrabajadores del IMSS (RJP) & 251567 \\
ISSSTE & 962075 \\
$\quad$ Cuentas individuales & 16710 \\
$\quad$ Décimo transitorio & 408398 \\
Ley abrogada & 536967 \\
SEDESOL (PAM) & 5487664 \\
Gobierno de la Ciudad de México (pensión & 480000 \\
alimentaria para mayores de sesenta y ocho & \\
años) & \\
Total & 10518703 \\
\hline
\end{tabular}

Fuentes: Instituto Mexicano del Seguro Social (2015), Instituto de Seguridad y Servicios Sociales de los Trabajadores del Estado (2016), Gobierno del Distrito Federal (2015) y Secretaría de Desarrollo Social (2015).

Aun con toda la importancia que en verdad tienen, los planes asistenciales son un complemento que no soluciona el problema de fondo. En México, el acceso a una pensión por retiro está limitado por las características eminentemente contributivas de los esquemas de pensiones. SEDESOL estima en 6.3 millones el número de adultos mayores de sesenta años o más que no perciben ingresos por jubilación o pensión contributiva (Secretaría de Desarrollo Social, 2015).

\section{Descapitalización de los institutos de seguridad social}

La situación financiera del IMSS y el ISSSTE no deja espacio al optimismo. En su último informe al Ejecutivo y al Congreso, el IMSS reconoció que dos de los cinco seguros que ofrece son deficitarios, y advirtió que el instituto podría caer en la insuficiencia financiera en 2019 (Instituto 
Mexicano del Seguro Social, 2016). El tono del informe fue, sin embargo, triunfalista, y presumió como un logro que el instituto no hubiera caído en la insolvencia financiera en 2014, como lo había pronosticado en su informe de 2012, ni tampoco en 2016, como reconoció que podría pasar en su informe de 2014 (Instituto Mexicano del Seguro Social, 2016, p. 24).

El ISSSTE luce más sólido, pero cinco de sus nueve fondos operan con déficit (Instituto de Seguridad y Servicios Sociales de los Trabajadores del Estado, 2016). Durante 2015, el Gobierno federal transfirió 13070 millones de pesos al ISSSTE para cubrir estos faltantes (Instituto de Seguridad y Servicios Sociales de los Trabajadores del Estado, 2016).

\section{I Las muchas causas}

La precaria situación financiera de los principales institutos de seguridad social del país es el resultado de una combinación de varios factores. Sin duda, la causa principal es la falta de crecimiento económico. Las finanzas de los institutos fueron severamente golpeadas por las crisis económicas de 1982, 1995 y 2008. También han resentido las políticas económicas que desde 1982 aplica el Gobierno mexicano, especialmente la decisión de usar los salarios como ancla de la inflación por el desplome de los salarios reales que provocó esta medida.

Hasta 1982, y desde la creación del sistema de seguridad social en 1943, la seguridad social mexicana se desarrolló gracias a la rápida expansión del empleo formal (Lomelí, 2001), pero "la crisis frenó el crecimiento del número de los asegurados, mermó los recursos de los organismos de la seguridad social y redujo el poder adquisitivo de las pensiones" (Lomelí, 2001, p. 203). Después de 1982, la economía mexicana ha tenido grandes dificultades para generar el empleo formal en la cantidad necesaria y la calidad suficiente, lo que ha traído como consecuencia el desmesurado 
crecimiento de las actividades informales, que por definición incumplen con el pago de la seguridad social (Lomelí, 2001).

La doble transición demográfica y epidemiológica que experimenta México, como otros países del mundo, es otra causa de los problemas financieros del IMSS y el ISSSTE. La primera transición, la demográfica, "aumentó la relación entre el número de pensionados y el de trabajadores en activo, lo que provocó que el modelo de reparto en el que estaba basado el sistema de pensiones se acercara a la insolvencia" (Lomelí, 2001, p. 203). La segunda transición, por su parte, aumentó la incidencia de las enfermedades crónicas, cuya atención es más costosa y amenaza con disparar los gastos en salud, lastrando aún más los seguros públicos de salud, que tradicionalmente han operado con déficit (González Block, 2015).

Que el Gobierno hubiera privado a los institutos del manejo de los fondos de pensiones también golpeó sus finanzas, "cortando el flujo de capital de interés de las cuentas de los pensionados que con anterioridad la seguridad social manejaba para su funcionamiento global" (Casas y Rodríguez, 2014, p. 90). Hasta 1997, cuando entró en vigor la reforma a la ley del IMSS de 1995, los remanentes del fondo de pensiones se aplicaron para cubrir el permanente déficit del seguro de enfermedades y maternidad (SEM) del IMSS (García Nieto, 2004). Sin estos fondos, que ahora se canalizan a las AFORES, el incurable déficit del SEM ha comenzado a cubrirse con transferencias del Gobierno federal (Instituto Mexicano del Seguro Social, 2016).

A todo lo anterior se suma la amplia y difundida evasión en el pago de cuotas por parte de los patrones. "Desafortunadamente, la evasión de la seguridad social es masiva en el país" (Levy, 2011, pp. 460-461). 


\subsection{El costo laboral de los institutos de seguridad social}

En los estados de actividades de los institutos de seguridad social sobresalen los pasivos laborales. En el caso del IMSS, el costo neto del periodo (costo derivado de la relación laboral atribuible al año de valuación) sumaba 779924 millones de pesos en el ejercicio anual concluido el 31 de diciembre de 2015, de los cuales 713955 millones de pesos correspondían al costo por beneficios a empleados (el ya mencionado Régimen de Jubicaciones y Pensiones, RJP), una cifra que equivale a más del doble de los ingresos del instituto en el mismo año, valuados en 354102 millones de pesos (Instituto Mexicano del Seguro Social, 2016, p. 15).

Los médicos, enfermeras y demás empleados del IMSS y el ISSSTE gozan de planes de pensiones relativamente generosos, pactados en los contratos colectivos de trabajo celebrados por los institutos con el Sindicato Nacional de Trabajadores del IMSS (SNTIMSS) y con el Sindicato Nacional de Trabajadores del ISSSTE (SNTISSSTE), respectivamente. Se trata de regímenes creados y constantemente mejorados por la presión de los sindicatos, que en cada renegociación exitosa del contrato obtuvieron más beneficios para sus representados.

En el caso del IMSS, las pensiones se reglamentan en la cláusula 110 del contrato colectivo de trabajo, que establece el RJP, por acuerdo de convenio del 7 de octubre de 1966. El RJP es un plan jubilatorio adicional al establecido en la ley del IMSS. Con el paso de los años, la cláusula 110 fue incorporando, en cada negociación exitosa, diversas aportaciones. Lo hizo en 1968, 1969, 1982 y 1988. En 1988, la cláusula 110 del contrato del IMSS autorizaba jubilarse a los trabajadores con treinta años de servicio en el instituto, sin límite de edad, con la cuantía máxima que otorgaba el régimen, muy superior a lo establecido en la ley del IMSS (García Nieto, 2004, p. 54). Sin embargo, una vez iniciada la transformación de los planes de pensiones en cuentas indi- 
vidualizadas de ahorro, el RJP fue considerado un objetivo a reformar. El IMSS advirtió al sindicato su falta de recursos para solventar el RJP.

Aunque García Nieto calcula que el RJP se quedó sin fondos suficientes desde 1994, advierte un uso tramposo de las cifras por parte del propio instituto, que incluye en ellas una prima de antigüedad que establece la Ley Federal del Trabajo, al margen del régimen contractual de jubilación y pensiones (García Nieto, 2004). El RJP se mantuvo en pie, sin cambios, hasta el 2004. En ese año, el Congreso reformó la Ley del Seguro Social y de esa forma alteró las bases legales del RJP. En 2005 y 2008, el IMSS y el sindicato de sus trabajadores firmaron convenios que redujeron el costo del RJP (Instituto Mexicano del Seguro Social, 2016, p. 23). En cualquier caso, los pasivos laborales siguen lastrando la suficiencia financiera del instituto. El IMSS reconoce que “[...] el crecimiento de la nómina del régimen de jubilaciones y pensiones sigue siendo el mayor reto que enfrenta el instituto" (Instituto Mexicano del Seguro Social, 2016, p. 23).

\subsection{Los seguros públicos de salud}

Despojados de los fondos de pensiones y faltos de un seguro de desempleo, la función que desempeñan los institutos de seguridad social se concentra, básicamente, en proteger la salud de sus asegurados. En realidad, el IMSS y el ISSSTE, junto con el llamado Seguro Popular, un programa asistencial diseñado para personas sin seguridad social, son las mayores "instituciones de protección en salud" en México (Instituto Mexicano del Seguro Social, 2016, p. 4). 
Tabla 6. Cobertura de las instituciones de protección

en salud al 31 de diciembre de 2015

$\begin{array}{lcc}\text { Instituciones } & \begin{array}{l}\text { Número de derechohabi- } \\ \text { entes/afiliados }\end{array} & \begin{array}{l}\text { Porcen- } \\ \text { taje de la } \\ \text { población }\end{array} \\ \text { IMSS } & 74032437 & 60.9 \\ \text { ISSSTE } & 12973731 & 10.8 \\ \text { Seguro Popular } & 57105622 & 47.4 \\ \text { PEMEX, Secretaría de la Defensa } & 1893946 & 1.6 \\ \text { Nacional, Secretaría de Marina- } & & \\ \text { Armada de México } & 2189514 & 1.8 \\ \text { Instituciones privadas } & 1824595 & 1.5 \\ \text { Otras instituciones } & \end{array}$

Nota: la suma de los porcentajes supera el $100 \%$ porque una parte de los asegurados tiene doble o triple registro.

Fuente: Instituto Mexicano del Seguro Social (2016).

Pese al tamaño relativo de la población que atienden, los institutos de seguridad social no reciben los recursos necesarios para la correcta operación de sus seguros de salud. En el caso del IMSS, las contribuciones de obreros, patrones y Gobierno al SEM se fijaron desde su creación en un monto notoriamente insuficiente para su operación (García Nieto, 2004). ${ }^{5}$ García Nieto ha reconstruido una serie estadística de cuarenta años con los resultados financieros del SEM, desde 1963, primer año con información disponible, hasta

5. En 1995, el entonces presidente Ernesto Zedillo reconoció el permanente déficit del SEM en la exposición de motivos de su iniciativa de reforma a la ley del IMSS de ese año, y lo explicó como producto de un mal cálculo: "El instituto [...] ha enfrentado crónicamente una insuficiencia financiera en el ramo de enfermedades y maternidad. Al ser creado este ramo en 1943, su cuota fue calculada sólo para dar protección al trabajador, aunque en una decisión favorable para la seguridad social, desde un principio se protegió a los familiares directos generándose así su desfinanciamiento" (Zedillo, 1995, p. 14). Un dato que confirma que la cuota de este seguro fue calculada considerando únicamente a los trabajadores lo aportan Casas y Rodríguez (20I4), quienes apuntan que "en un principio, el IMSS sólo atendía al trabajador; fue hasta 1949 que se extendió la seguridad social a toda la familia" (p. 90). 
el 2002. En este periodo, el SEM registró un déficit de operación en treinta y cinco años (García Nieto, 2004, pp. 78-79). Datos más recientes confirman que su déficit tiende a ser permanente (Instituto Mexicano del Seguro Social, 2016). Lo mismo sucede con el seguro de salud (SS) del ISSSTE (Instituto de Seguridad y Servicios Sociales de los Trabajadores del Estado, 2016).

Tabla 7. Seguros de salud IMSS e ISSSTE.

Resultados de operación (cifras en millones de pesos)

\begin{tabular}{lrrrrr} 
& 2011 & 2012 & 2013 & 2014 & 2015 \\
IMSS / SEM & & & & & \\
Total de ingresos & 187679 & 207924 & 216247 & 230048 & 243735 \\
Total de egresos & 861560 & 840148 & 844676 & 892656 & 915043 \\
Resultados & -673881 & -632224 & -628429 & -662608 & -671307 \\
ISSSTE / SS & & & & & \\
Total de ingresos & 44143 & 47017 & 49378 & 52025 & 55085 \\
Total de egresos & 52609 & 49489 & 51718 & 61435 & 63057 \\
Resultados & -8466 & -2472 & -2340 & -9410 & -7972 \\
\hline
\end{tabular}

Nota: los ingresos de ambos seguros se integran con cuotas obreropatronales, aportaciones del Gobierno federal y otros ingresos. Los egresos del SEM incluyen el costo neto del periodo, que se integra por los rubros de pagos a pensiones y jubilados IMSS, y el costo por beneficios a empleados del RJP. Los egresos del SS incluyen reservas.

Fuentes: Instituto Mexicano del Seguro Social (2012, p. 87; 2013, p. 89; 2014, p. 107; 2015, p. 122; 2016, p. 121) e Instituto de Seguridad y Servicios Sociales de los Trabajadores del Estado (2016, pp. 85-86).

El IMSS calcula que de cumplirse sus proyecciones para el periodo 2015-2050, el SEM podría acumular en treinta y cinco años un déficit equivalente al 12\% del PIB de 2015 (Instituto Mexicano del Seguro Social, 2015, p. 43). 


\section{Sistema universal de seguridad social}

Universalizar la seguridad social fue una de las promesas de campaña del actual presidente de la república, Enrique Peña Nieto (2012-2018), y uno de los acuerdos que firmó con los representantes de los principales partidos políticos el 2 de diciembre de 2012, en el llamado Pacto por México. ${ }^{6}$ El modelo de universalidad propuesto por los firmantes de este pacto es el de un conjunto de cuatro beneficios sociales procurados por el Estado: acceso universal a los servicios de salud, pensión universal, seguro de desempleo para trabajadores del sector privado y seguro de vida para jefas de familia.

Se ha dicho que la inspiración del modelo delineado en el pacto es la propuesta que formulara años antes Santiago Levy, exdirector del IMSS y funcionario del Banco Mundial (Flores, 2013). Levy, efectivamente, propuso otorgar la seguridad social a todos los trabajadores, pero su propuesta, a diferencia de la propuesta del Pacto por México, se basa en suprimir las cuotas obreros-patronales y remplazarlas por un impuesto general (Levy, 2011 y 2012). Pagar la seguridad social con impuestos generales evitaría discriminar entre derechohabientes (trabajadores formales y sus familias) y población abierta (trabajadores informales y sus familias), tal como ahora hacen los institutos de seguridad social. "No podemos hablar con seriedad de la universalización de un derecho, si no hablamos al mismo tiempo de una fuente de

6. El acuerdo I.I del Pacto por México dice: "Seguridad social universal.Se creará una red de protección social que garantice el acceso al derecho a la salud y otorgue a cualquier mexicano, independientemente de su condición social o estatus laboral, un conjunto de beneficios sociales procurados por el Estado a través de un sistema de seguridad social universal que incorpore lo siguiente: acceso universal a los servicios de salud; [...] pensión para los adultos mayores de sesenta y cinco años que no cuenten con un sistema de ahorro para el retiro o pensión del IMSS - ISSSTE; [...] seguro de desempleo; [...] [y] seguro de vida para jefas de familia" (Peña Nieto, Zambrano, Díaz, y Madero, 20I2, pp. 2-3). 
financiamiento que no discrimine entre los que disfrutan de ese derecho" (Levy, 2011, p. 468). Las cuotas obreropatronales, argumenta Levy, constituyen un "impuesto a la formalidad", por lo que eliminarlas remueve obstáculos a la plena formalización de la economía.

Por razones similares, agrega Levy, también debería cortarse el "subsidio a la informalidad" que, según el autor, constituyen los programas sociales asistenciales para personas sin seguridad social, los que por otra parte ya no tendrían razón de ser en un sistema universal de seguridad social (Levy, 2011). En palabras de Levy,

mi propuesta es establecer un impuesto al consumo, similar al impuesto al valor agregado, pero destinar específicamente los recursos generados por ese impuesto (o, mejor dicho, contribución) a garantizar los derechos de seguridad social de todos los trabajadores, con total independencia de su condición laboral (y, por supuesto, género o lugar de trabajo) (Levy, 20I I, p. 468).

Diferente o no de su supuesta inspiración, el sistema universal delineado en el Pacto por México plantea de cualquier modo una remodelación profunda del andamiaje institucional, y especialmente del de los institutos de seguridad social. En el tema de la salud, por ejemplo, se plantea

impulsar dos principios de reorganización del sector salud: el de la portabilidad de las coberturas[,] para que todos los asegurados puedan ser atendidos en cualquiera de los sistemas públicos de salud a su elección;y la convergencia de los sistemas[,] para igualar gradualmente la cobertura y calidad de todos ellos (Peña Nieto, Zambrano, Díaz,y Madero, 20I2, p. 2). ${ }^{7}$

7. La propuesta, sin embargo, coincide ampliamente con los grupos de interés del sector salud que impulsan una agenda de cambios pro consenso de Washington. Fundación Mexicana para la Salud (Funsalud), la fundación privada en temas de salud más influyente de México, no tuvo dificultades en hacer coincidir con esta propuesta su propia agenda de cambios para el sector de su interés. En la presentación de su propuesta de universalizar el sistema de salud, Funsalud afirma que 
Esta propuesta, no obstante, no prosperó. El 8 de septiembre de 2013, el Ejecutivo envió al Congreso de la Unión, como parte de su reforma hacendaria, una iniciativa de reforma a los artículos 4, 7 y 123 de la constitución para reconocer el derecho de los adultos mayores a una pensión universal y el derecho de los trabajadores del sector privado al seguro de desempleo (Monroy, 2014; Galicia, 2014). Sin embargo, hasta ahora la iniciativa no ha logrado completar su trámite. La Cámara de Diputados aprobó ya estas reformas constitucionales y las envió al Senado para su discusión, pero los senadores no las han ratificado ni enviado a los Congresos de los estados para su análisis y aprobación. Inicialmente, esto no detuvo a los diputados. Sin esperar la conclusión del trámite de reforma constitucional, la Cámara de Diputados aprobó y envió de igual manera al Senado la ley secundaria que establece "un ingreso mínimo de sobrevivencia para los adultos mayores", denominada Ley de Pensión Universal, y las modificaciones a la Ley del Seguro Social necesarias para establecer el seguro de desempleo (Monroy, 2014; Galicia, 2014; Bensusán, 2014).

Como sucedió con la reforma constitucional, la Ley de Pensión Universal y las modificaciones a la Ley del Seguro Social aguardan turno para su discusión y eventual aprobación. Los principales obstáculos que estas reformas encontraron en su camino se relacionan con los montos establecidos, considerados por sus críticos como notoriamente insuficientes. En el caso de la pensión universal, se estableció un monto de MXN 1092.00 mensuales, que corresponde a la línea de bienestar mínimo (LMB) definida por el Consejo

\footnotetext{
"la propuesta de reforma del sistema nacional de salud que presenta Funsalud converge con el planteamiento de un sistema de seguridad social universal, y se inscribe en uno de sus cuatro elementos fundamentales: acceso efectivo a los servicios de salud, pensión para la vejez, seguro de desempleo y seguro de riesgos de trabajo" (Fundación Mexicana para la Salud, 20I3, p. E5). No obstante, nótese que en la enumeración de los componentes del sistema de seguridad social universal, Funsalud excluye seguros de vida para jefas de familia e incluye seguros de riesgos de trabajo.
} 
Nacional de Evaluación de la Política de Desarrollo Social (Coneval) como el valor monetario mensual de una canasta alimentaria básica, y que sería actualizado anualmente conforme al INPC (Monroy, 2014).

Con relación al seguro de desempleo, las modificaciones a la Ley del Seguro Social pendientes de aprobación establecen un máximo de seis pagos mensuales, divididos en dos etapas: la primera etapa comprende los dos primeros pagos, por un monto correspondiente al $60 \%$ del salario promedio declarado en las últimas veinticuatro cotizaciones para el primer pago y de 50\% en el segundo pago; en la segunda etapa, para los cuatro pagos restantes, el monto equivale al 40\% del salario promedio. También ha sido muy criticada la decisión de los diputados de fondear este nuevo seguro con la subcuenta de vivienda de los trabajadores (Bensusán, 2014).

Es probable que estas iniciativas ya no serán discutidas durante el Gobierno del actual presidente, al que ya sólo le quedan algunos meses, y como no es común que una legislatura recoja una iniciativa congelada en la anterior, se puede suponer que ambas iniciativas ya no serán aprobadas, no al menos en sus actuales términos.

Después de la privatización de los fondos de pensiones, la descapitalización de los institutos de seguridad social y la promesa fallida de un seguro de desempleo, queda muy poco del sistema mexicano de seguridad social. Los institutos conservan la administración de varios seguros, pero padecen de una falta crónica de recursos. En la opinión de los autores del presente trabajo, el futuro de estos institutos depende del rumbo que tome la universalización de los servicios de salud. Hasta ahora, las reformas prouniversalización de los servicios de salud que empuja el Gobierno de México desde 2003 se han concentrado en la ampliación del Seguro Popular, un seguro prácticamente gratuito, diseñado para la población sin seguridad social, pero se espera que pronto las reformas alcancen 
a los seguros de salud del IMSS e ISSSTE, de los que, ya se dijo, depende la mayoría de los mexicanos.

Las principales propuestas que se discuten hoy en día recomiendan transformar y reorganizar estos institutos para integrar, homogenizar y estandarizar sus procedimientos médicos como paso previo a la plena portabilidad de la cobertura (que el afiliado de un instituto se atienda en otro) y la convergencia de los sistemas contributivos y no contributivos (convergencia medida en servicios equiparables). En este punto no hay nada escrito, pero la propuesta con más apoyo, incluyendo el de los tres últimos presidentes de la república, es la que impulsa Funsalud, que recomienda dividir a cada instituto en dos o más organismos para separar la prestación de los servicios de salud de sus fuentes de financiamiento, a los que por otra parte tendrían acceso los médicos y hospitales privados (Fundación Mexicana para la Salud, 2013). El debate apenas comienza, pero es obvio que el foco de las reformas al sistema de seguridad social se ha trasladado de las pensiones a los seguros de salud.

\section{Conclusiones}

Luego de treinta años de ajustes y recortes, el sistema mexicano de seguridad social luce igual o peor que antes. Considerando los sacrificios exigidos y los beneficios prometidos, los resultados de las reformas pro consenso de Washington son decepcionantes. La situación financiera de los institutos de seguridad social sigue siendo precaria, ${ }^{8}$ y su cobertura limitada. No obstante, sus responsables siguen actuando como si todo se resolviera insistiendo

8. Es sintomático que el director general del mayor de estos institutos presuma que su viabilidad financiera es ahora de tres años:"El tema más relevante para el futuro del IMSS es la viabilidad financiera, en 2012 estaba garantizada hasta 2014 , en 20 I6[,] el año pasado[,] anunciamos que estaba garantizada hasta el 20 I 9, con el esfuerzo de todos [...], hoy podemos anunciar que la viabilidad financiera está garantizada hasta el 2020, ya trascendió esta administración” (Rivera, 2017, p. 7). 
en las mismas medidas: elevar las contribuciones de los beneficiarios, reducir los beneficios, endurecer los criterios de acceso y transferir al mercado la operación del sistema, todas recetas amargas que poco han ayudado a resolver el problema.

Basta comparar los beneficios que los reformadores prometieron cuando presentaron la privatización de los planes pensionarios como un cambio indispensable, inevitable y urgente con los reducidos montos de las pensiones que ahora se pagan y la limitada cobertura de los actuales sistemas privados de ahorro individual para el retiro. A cambio de muy poco, se quebró el principio de solidaridad intergeneracional.

Los supuestos ahorros que el Estado esperaba del nuevo esquema de pensiones se evaporaron con el alto costo fiscal de la transición y las grandes y continuas transferencias del Gobierno federal a los institutos de seguridad social para compensar la pérdida de estos fondos. Este costo y estas transferencias apenas se discutieron cuando se preparaban las reformas, y aún ahora no se reconocen como una falla o un error de las políticas instrumentadas a lo largo de estos años. Al contrario: se asumen como una razón más para continuar profundizando en el despojo de estos fondos. El monto de fondos manejados por las AFORES, la métrica que los responsables del sistema reformado utilizan para medir su éxito, es la evidencia del verdadero propósito del cambio de esquema.

Las propuestas para universalizar la protección social que ahora se discuten están lastradas por la misma fe ciega en el mercado. Ciertamente, el Estado de hoy en día no tiene la capacidad financiera del pasado, pero esa no es realmente la causa sino el pretexto de la orientación privatizadora de las reformas al sistema. No es que la falta de recursos para fondear el sistema no sea un problema. Lo es, y quizá sea, en estos momentos, el mayor de los problemas del sistema, 
pero muy probablemente las reformas que se requieren sean otras. En este punto, baste recordar, como se ha dicho ya, que el problema mayor de los institutos de seguridad social está en los seguros de salud que operan con grandes déficits por la renuncia del Gobierno y la oposición de los patrones a fijar una cuota que cubra totalmente sus costos de operación.

Se ha perdido un tiempo precioso desmantelando lo que bien o mal construyeron las generaciones del desarrollo estabilizador. Ahora quizá sea muy tarde para volver atrás, pero aún es tiempo de evitar caer en el mismo error de no discutir lo suficiente las posibles reformas de un sistema de seguridad social como el mexicano, tan grande, tan complejo y tan importante para el común de la gente.

Para un debate de esta clase, la pregunta que importa responder en este momento es qué clase de universalización quiere, necesita y puede costear el país. En el caso de la salud, la Organización Mundial de la Salud (OMS) ha señalado que la universalización es un proceso gradual que corre por tres caminos paralelos: amplitud (porcentaje de la población con acceso al sistema), profundidad (proporción de tratamientos y medicamentos cubiertos) y gratuidad (porcentaje del costo libre de pago). Hasta ahora, la coalición gobernante parece inclinarse por la máxima cobertura posible con beneficios muy limitados, incluso insuficientes, para los más pobres, y por los beneficios adicionales que cada persona pueda pagar para el resto de la población. ¿Es esto lo que requiere el país? ¿No podría hacerse algo mejor para todos?

Auditoría Superior de la Federación (20l4). Evaluación número I 203. Evaluación de la política pública de pensiones y jubilaciones. México: Cámara de Diputados. Recuperado de: http://www.asf.gob.mx/Trans/Informes/IR20I3i/ Documentos/Auditorias/2013_I203_a.pdf 
Bibliografía

Bensusán, G. (20 I4). El seguro de desempleo en México. Opciones, características, ventajas y desventajas. Santiago de Chile: CEPAL.

Casas, D., y Rodríguez, A. (20I4). "La caída de la seguridad social en México: caso IMSS”. Archivos de investigación materno infantil, VI(2), 85-94.

Comisión Nacional del Sistema de Ahorro para el Retiro (20I4). Informe trimestral al H. Congreso de la Unión sobre la situación del SAR -4to trimestre 2013. Recuperado de: https://www.gob.mx/cms/uploads/attachment/ file/6I268/20I3_20_trimestre_4.pdf

Flores, G. (I0 de septiembre de 20I3). “¿Entiende bien el Gobierno la seguridad social 'universal'?". Nexos. Recuperado de: http://redaccion.nexos.com.mx/?p=5648

Fundación Mexicana para la Salud (2013). "Universalidad de los servicios de salud en México". Salud pública de México, 55, EI-E64. Recuperado de: http://saludpublica. $\mathrm{mx} /$ index.php/spm/article/view/8759/I I I05

Galicia,P.(20I4).“El difícil camino para la creación del seguro de desempleo en México". Revista latinoamericana de derecho social, (I8), I6I-I65.

García Nieto, U. (coord.) (2004). El Instituto Mexicano del Seguro Social y la crisis financiera del esquema de jubilaciones y pensiones de sus trabajadores. México-Bonn: Fundación Friedrich Ebert.

Gobierno del Distrito Federal (2015). Programa Pensión Alimentaria para Adultos Mayores de 68 años. Evaluación interna 2015. México: Secretaría de Desarrollo Social, Instituto para la Atención de Adultos Mayores. Recuperado de: http://www.adultomayor.cdmx.gob.mx/images/ pdf/EVALUACIONINTERNAIAAMDF.pdf

González Block, M.A. (coord.) (20I5). Diagnóstico de los retos al sistema nacional de salud universal. México: Funsalud, Consejo Promotor de Competitividad y Salud. 
Ham, R. (2000). "Sistemas de pensiones y perspectivas de la Bibliografía seguridad social”. Estudios demográficos y urbanos, I5(3), 613-639.

Instituto de Seguridad y Servicios Sociales de los Trabajadores del Estado (27 de diciembre de 1983). “Ley del Instituto de Seguridad y Servicios Sociales de los Trabajadores del Estado". Diario Oficial de la Federación, pp. I-29 [segunda sección]. Recuperado de: http://dof.gob. $\mathrm{mx} /$ index. . php? year $=1983 \&$ month $=\mid 2 \&$ day $=27$

Instituto de Seguridad y Servicios Sociales de los Trabajadores del Estado (3I de marzo de 2007). “Ley del Instituto de Seguridad y Servicios Sociales de los Trabajadores del Estado”. Diario Oficial de la Federación, Pp. I-64. Recuperado de: http://dof.gob.mx/index.php?year=2007\&month $=03 \&$ day $=31$

Instituto de Seguridad y Servicios Sociales de los Trabajadores del Estado (2016). Informe financiero y actuarial (IFA) 20/6. México: ISSSTE. Recuperado de: http://www.issste. gob.mx/images/downloads/instituto/quienes-somos/ ifa_2016.pdf

Instituto Mexicano del Seguro Social (24 de febrero de 1992). "Decreto que reforma y adiciona diversas disposiciones de la Ley del Seguro Social y de la Ley del Impuesto Sobre la Renta". Diario Oficial de la Federación, pp. 29-34 [segunda sección]. Recuperado de: http://www.dof.gob. $\mathrm{mx} /$ index.php? year $=1$ 992\&month $=02 \&$ day $=24$

Instituto Mexicano del Seguro Social (2I de diciembre de 1995). "Ley del Seguro Social”. Diario Oficial de la Federación, pp. 25-63. Recuperado de: http://www.dof.gob. $\mathrm{mx} /$ index.php? year $=\mid$ 995\&month $=\mid 2 \&$ day $=2 \mid$

Instituto Mexicano del Seguro Social (2012). Informe al Ejecutivo federal y al Congreso de la Unión sobre la situación financiera y los riesgos del Instituto Mexicano del Seguro Social 20 I I-20 I 2. México: IMSS. Recuperado de: https:// doczz.es/doc/12172/informe-art\%C3\%ADculo-Iss-273 
Bibliografía

Instituto Mexicano del Seguro Social (2013). Informe al Ejecutivo federal y al Congreso de la Unión sobre la situación financiera y los riesgos del Instituto Mexicano del Seguro Social 20/2-20/3. México: IMSs. Recuperado de: http://www. imss.gob.mx/sites/all/statics/pdf/informes/20122013/ InformeCompleto.pdf

Instituto Mexicano del Seguro Social (20I4). Informe al Ejecutivo federal y al Congreso de la Unión sobre la situación financiera y los riesgos del Instituto Mexicano del Seguro Social 20/3-20/4. México: IMSS. Recuperado de: http://www. imss.gob.mx/sites/all/statics/pdf/informes/20 I320|4/2I_ InformeCompleto.pdf

Instituto Mexicano del Seguro Social (20I5). Informe al Ejecutivo federal y al Congreso de la Unión sobre la situación financiera y los riesgos del Instituto Mexicano del Seguro Social 2014-20/5. México: IMSS. Recuperado de: http://www. imss.gob.mx/sites/all/statics/pdf/informes/20 I420I5/2I InformeCompleto.pdf

Instituto Mexicano del Seguro Social (2016). Informe al Ejecutivo federal y al Congreso de la Unión sobre la situación financiera y los riesgos del Instituto Mexicano del Seguro Social 20/5-20/6. México: IMSS. Recuperado de: http://www. imss.gob.mx/sites/all/statics/pdf/informes/20I520I6/2IInformeCompleto.pdf

Instituto Nacional de Estadística y Geografía (2010). Censo general de población y vivienda 2010. México: INEGI. Recuperado de: http://www3.inegi.org.mx/sistemas/TabuladosBasicos/Default.aspx?c=27302

Instituto Nacional de Estadística y Geografía (20 I4). Resultados de la Encuesta nacional de ocupación y empleo. Cifras durante el cuarto trimestre 2013. México: INEGI. Recuperado de: http://www.inegi.org.mx/saladeprensa/boletines/20 I4/ enoe/enoe20I4_02.pdf

Levy, S. (20II). "¿Universalización de la salud o seguridad social?”. Gaceta médica de México, (147), 455-468. 
Levy, S. (20I2). "Seguridad social universal: un camino para Bibliografía México”. Nexos, (4I9), 37-4I.

Lomelí, L. (200I). "Perspectivas de la seguridad social en México”. Revista mexicana de sociología, 63(I), 203-220.

Monroy, N. (20I4). "La reforma constitucional y la ley de pensión universal”. Revista latinoamericana de derecho social, (I8), I67-I73.

Morales, M. A. (2007). "Nueva ley del ISSSTE y pensiones de retiro". Revista latinoamericana de derecho social, (5), 263268.

Murillo, S.,y Venegas, F. (20I I).“Cobertura de los sistemas de pensiones y factores asociados al acceso a una pensión de jubilación en México". Papeles de población, 17(67), 209-250.

Organización para la Cooperación y el Desarrollo Económicos (2016). Estudio de la OCDE sobre los sistemas de pensiones: México. México: CONSAR. Recuperado de: https://www.gob.mx/cms/uploads/attachment/file/61968/ sistema_de_pensiones_2016.pdf

Peña Nieto, E., Zambrano, J., Díaz, M. C., y Madero, G. (20I2). Pacto por México. Recuperado de: https://embamex.sre. gob.mx/bolivia/images/pdf/REFORMAS/pacto_por_ mexico.pdf

Rivera,A. (I5 de noviembre de 20 I7). “Garantizada viabilidad financiera del IMSS hasta 2020: Mikel Arriola”. El Universal, p. 7.

Secretaría de Desarrollo Social (20I5). Programa de Subsidio del Ramo Administrativo 20, Desarrollo Social: cuarto informe trimestral 20 14. México:Dirección General de Seguimiento de la SEDESOL. Recuperado de: http://www.sedesol.gob. $\mathrm{mx} /$ work/models/SEDESOL/Transparencia/InformesPresupuestoEjercido/Cuarto_Informe_Trimestral_20I4.pdf Secretaría del Trabajo y Previsión Social (1 2 de marzo de 1973). “Ley del Seguro Social”. Diario Oficial de la Fede- 
Bibliografía ración, pp. 12-38. Recuperado de: http://www.dof.gob. $\mathrm{mx} /$ index.php? year $=1973 \&$ month $=03 \&$ day $=12$

Solís, F., y Villagómez, A. (1999). “Las pensiones”, en F. Solís, y A.Villagómez (comps.), La seguridad social en México (pp. 103-159). México: Fondo de Cultura Económica.

Valencia, E.(2016).“Viejas y recientes reformas: trayectoria de un mismo ciclo”, en E.Valencia, y G. Ordóñez (coords.), Nueva ronda de reformas estructurales en México, ¿nuevas políticas sociales? (Pp. 13-30). Tijuana: El Colegio de la Frontera Norte.

Valencia, E., y Ordóñez, G. (coords.) (2016). Nueva ronda de reformas estructurales en México, ¿nuevas políticas sociales? Tijuana: El Colegio de la Frontera Norte.

Villagómez, A. (20I5)."México: un esquema multipolar fragmentado", en R.Acuña (coord.), Cómo fortalecer los sistemas de pensiones latinoamericanos (pp. 293-362). Santiago de Chile: SURA Asset Management.

Zedillo, E. (1995). Decreto de Ley del Seguro Social del 21 de diciembre de 1995. México: Instituto Mexicano del Seguro Social. 